\title{
Matrine inhibits the invasive properties of human glioma cells by regulating epithelial-to-mesenchymal transition
}

\author{
ZHONGWEI WANG ${ }^{1}$, YI WU ${ }^{2}$, YALI WANG ${ }^{1}$, YINGYING JIN ${ }^{1}$, \\ XIULONG MA ${ }^{1}$, YANG ZHANG ${ }^{1}$ and HONGTAO REN ${ }^{1}$ \\ ${ }^{1}$ Department of Medical Oncology, The Second Affiliated Hospital, School of Medicine, Xi'an Jiaotong University, \\ Xi'an, Shaanxi 710004; ${ }^{2}$ Department of Pathology, Children's Hospital Affiliated to Soochow University, \\ Suzhou, Jiangsu 215003, P.R. China
}

Received April 11, 2014; Accepted November 7, 2014

DOI: $10.3892 / \mathrm{mmr} .2015 .3167$

\begin{abstract}
Matrine is reported to be effective in tumor therapies; however, the anti-metastatic effect and molecular mechanism(s) of matrine on glioma remain poorly understood. Therefore, the purpose of this study was to assess the effects of matrine on glioma and the associated mechanism(s). In the study, we demonstrated that matrine inhibited the proliferation of glioma cells. We also observed that matrine inhibited the migration and invasion of glioma cells at non-toxic concentrations. Matrine also decreased the expression of E-cadherin and increased the expression of $\mathrm{N}$-cadherin. These results suggest that the anti-metastatic effect of matrine may be correlated with epithelial-to-mesenchymal transition (EMT). Moreover, matrine could reduce the phosphorylation levels of p38 and AKT proteins. In conclusion, these results suggest matrine may be a potential alternative against invasive glioma cells via the p38 MAPK and AKT signaling-dependent inhibition of EMT.
\end{abstract}

\section{Introduction}

Malignant glioma is characterized by its highly invasive growth (1). Due to the rapid and invasive tumor growth, current treatments for malignant gliomas, including surgery, chemotherapy and radiation, have not been successful $(2,3)$. According to statistics, the majority of malignant gliomas are resistant to chemotherapeutic agents, and patients have a mean survival time of 12 months following diagnosis (4). Therefore, it is necessary to develop novel agents for combating malignant gliomas.

Metastasis involves multiple processes and various cytophysiological changes; among them, epithelial-to-mesen-

Correspondence to: Dr Hongtao Ren, Department of Medical Oncology, The Second Affiliated Hospital, School of Medicine, Xi'an Jiaotong University, 157 West Fifth Road, Xi'an, Shaanxi 710004, P.R. China

E-mail: tougaoyouxiang2014@163.com

Key words: matrine, glioma, invasion, epithelial-to-mesenchymal transition, p38, AKT chymal transition (EMT) is a key step. EMT involves the loss of epithelial markers, including E-cadherin. Concomitantly, the expression of a number of mesenchymal markers is increased, including $\mathrm{N}$-cadherin, vimentin and matrix metalloproteinase. Through EMT, cancer cells obtain enhanced motility, which enables metastasis.

Matrine, the molecular formula of which is $\mathrm{C}_{15} \mathrm{H}_{24} \mathrm{~N}_{2} \mathrm{O}$, is derived from Sophora species of plants and has a long history of use in traditional Chinese medicine to treat inflammation (5). Matrine has been shown to produce a wide range of pharmacological effects and has also been used in the treatment of cancer (5-8). However, there are no studies on the anti-metastatic effect of matrine on glioma. In the present study, we investigate the effects and mechanisms of matrine against glioma invasion.

\section{Materials and methods}

Reagents. Phosphate-buffered saline (PBS), dimethyl sulfoxide (DMSO), polycarbonate membrane filters, polylysine-coated slides, paraformaldehyde, 3,3'-diaminobenzidine, hematoxylin, lysis buffer and PVDF membranes were purchased from Shanghai Abcam Biological Products Co., Ltd (Shanghai, China). Fetal bovine serum (FBS), penicillin and streptomycin were purchased from Shanghai Worship Biological Technology Co., Ltd (Shanghai, China). Matrine was purchased from Shanghai Chuan Xiang Biological Technology Co., Ltd (Shanghai, China). Dulbecco's modified Eagle's medium (DMEM) was purchased from Invitrogen Life Technologies (Carlsbad, CA, USA). Anti-p38 (AB01165), anti-AKT (AB01313) and anti- $\beta$-actin (4967L) antibodies were purchased from Cell Signaling Technology, Inc. Anti-p-p38 (sc-166182) was purchased from Santa Cruz Biotechnology, Inc. (Dallas, TX, USA). Anti-E-cadherin (ab11512) and anti-N-cadherin (ab11339) antibodies were purchased from Shanghai Abcam Biological Products Co., Ltd (Shanghai, China). Anti-p-AKT (AP0056) was purchased from Shanghai Seebio Biotech, Inc. (Shanghai, China).

Cell culture. The human glioma cell lines U251MG and U87MG (obtained from a cell bank at the Fourth Military Medical University, Xi'an, China) were cultured in DMEM supplemented with $10 \% \mathrm{FBS}$. All cells were incubated at $37^{\circ} \mathrm{C}$ with $5 \% \mathrm{CO}_{2}$. 
Cell viability assays. Cell survival was assessed using standard 3-(4,5-dimethylthiazol-2-yl)-2,5-diphenyltetrazolium bromide (MTT) assay as previously described (9). Briefly, cells were plated in 96 -well culture plates $\left(3 \times 10^{4}\right.$ cells per well). The cells were treated with various concentrations of matrine. After $24 \mathrm{~h}$ incubation, the cells were washed twice with PBS and incubated with $5 \mathrm{mg} / \mathrm{ml}$ MTT (Sigma, St. Louis, MO, USA) for $4 \mathrm{~h}$. Following the incubation period, the cells were washed with PBS and then solubilized with DMSO. The optical density was read on an enzyme-linked immunosorbent assay plate reader (frequency, 3; Victor X2 Multilabel Plate reader, Perkin-Elmer, Waltham, MA, USA).

In vitro invasion and migration assays. The in vitro invasion and migration activity was measured according to the methods described previously (10). U87MG cells were pretreated with $0,0.2,0.4$ and $0.8 \mathrm{mg} / \mathrm{ml}$ matrine for $24 \mathrm{~h}$, then surviving cells were harvested and seeded to Transwell chambers (Corning, Inc., Corning, NY, USA) at $2 \times 10^{5}$ cells/well in serum-free medium and then incubated for $24 \mathrm{~h}$ at $37^{\circ} \mathrm{C}$. At the endpoint, the cells on the upper side of the inserts were completely removed by swabbing, while the cells on the bottom side of the filter were fixed, stained and measured. For the invasion assay, $50 \mu \mathrm{l}$ Matrigel $(25 \mu \mathrm{g} / \mathrm{ml}$; BD Biosciences, Bedford, MA, USA) was applied to $8-\mathrm{mm}$ pore size polycarbonate membrane filters.

Immunocytochemistry (ICC). ICC was performed as described previously (11); cells were seeded on polylysine-coated glass slides, cultured for 2 days, fixed in $4 \%$ paraformaldehyde, then incubated with primary antibody. Subsequently, sections were incubated with biotinylated secondary antibody and visualized with 3, 3'-diaminobenzidine; the nuclei were counter-stained with hematoxylin. Negative controls were prepared using the same procedure, but PBS was substituted for primary antibody.

Western blot analysis. Cells were suspended in lysis buffer (40 mmol/1 Tris- $\mathrm{HCl}, 1 \mathrm{mmol} / 1$ EDTA, $150 \mathrm{mmol} / 1 \mathrm{KCl}$, $100 \mathrm{mmol} / 1 \mathrm{NaVO}_{3}, 1 \%$ Triton X-100, 1 mmol/1 PMSF, pH 7.5), following treatment with various concentrations of matrine. The proteins were separated by $10 \%$ or $8 \%$ SDS-polyacrylamide gel electrophoresis and transferred onto PVDF membranes. The membranes were subsequently blocked in defatted milk at room temperature for $1 \mathrm{~h}$ and then incubated with antibodies against E-cadherin, $\mathrm{N}$-cadherin, p38, p-p38, AKT, $\mathrm{p}$-AKT or $\beta$-actin at $4^{\circ} \mathrm{C}$ overnight. The membranes were then incubated with a horseradish peroxidase goat anti-mouse or anti-rabbit IgG antibody for $1 \mathrm{~h}$ at room temperature. The bands were detected with an enhanced chemiluminescence kit (Amersham, ECL Plus, Freiburg, Germany) and exposed by autoradiography. The densitometric analysis was performed using Image $\mathbf{J}$ software (GE Healthcare, Buckinghamshire, $\mathrm{UK})$, and the results were expressed as arbitrary units.

Statistical analysis. All experiments were repeated three times. The statistical significance of differences throughout this study was analyzed by the one-way ANOVA test to compare differences between treatments, and followed up using Dunnett's multiple comparison post hoc test. All statistical tests and corresponding $\mathrm{P}$-values were two-sided. $\mathrm{P}<0.05$

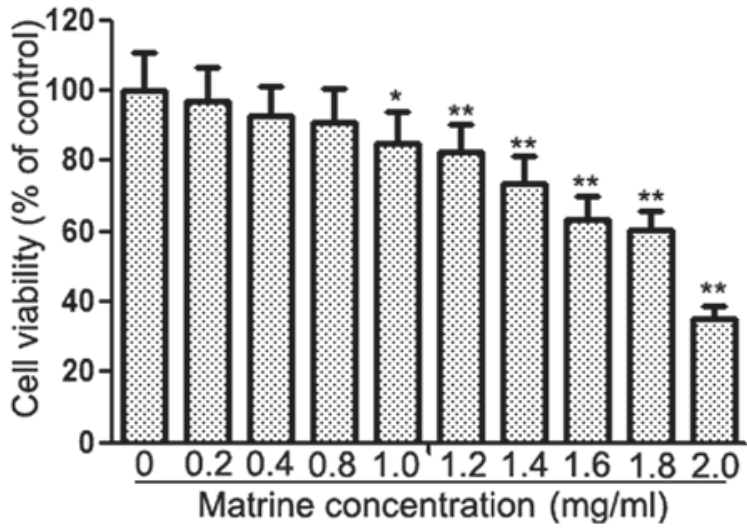

Figure 1. Cellular viability of U87MG cells treated with matrine. U87MG cells were treated with various concentrations of matrine, and after $24 \mathrm{~h}$ their viability was measured using an MTT assay. Values represent the means \pm SD of three independent experiments, each performed in triplicate. ${ }^{*} \mathrm{P}<0.05$ and ${ }^{* *} \mathrm{P}<0.01$ compared with control group.

was considered to indicate a statistically significant difference. Correlation analysis was performed using the Z-test.

\section{Results}

Matrine inhibits the proliferation of U87MG glioblastoma cells. The effects of matrine at various concentrations ( 0 to $2 \mathrm{mg} / \mathrm{ml}$ ) on the proliferation of U87MG cells are shown in Fig. 1. At $1 \mathrm{mg} / \mathrm{ml}$, matrine clearly inhibited the proliferation of U87MG cells, while at concentrations below $1 \mathrm{mg} / \mathrm{ml}$, the inhibition effect was not significant. Hence we selected a concentration range of matrine lower than this for all subsequent experiments.

Matrine inhibits the migration and invasion of U87MG cells. Fig. 2 demonstrates the effect of matrine on the migration and invasion of U87MG cells that were treated with $0,0.2$, 0.4 and $0.8 \mathrm{mg} / \mathrm{ml}$ matrine for $24 \mathrm{~h}$ in the cell migration and cell invasion assays. The results revealed that matrine reduced the invasion and migration of U87MG cells substantially in a concentration-dependent manner. Matrine also inhibited the invasion of U251MG (Fig. 3).

Matrine suppresses the expression of $N$-cadherin and increases the expression of E-cadherin. Cells were treated with $0,0.2,0.4$ and $0.8 \mathrm{mg} / \mathrm{ml}$ matrine for $24 \mathrm{~h}$ and then subjected to western blot analysis to test the expression of E-cadherin and N-cadherin in U87MG cells. Fig. 4A and B reveal that matrine significantly reduced the protein levels of $\mathrm{N}$-cadherin and increased the expression of E-cadherin in a concentration-dependent manner compared with the control group. Fig. 4C demonstrates the effect of matrine on the expression of E-cadherin and $\mathrm{N}$-cadherin. From the image, it can be observed that matrine increased the expression of E-cadherin and reduced the expression of N-cadherin. Matrine also increased the expression of E-cadherin and reduced the expression of N-cadherin in U251MG cells (Fig. 5).

p38 MAPK and AKT are involved in the anti-metastatic mechanism of matrine. In human glioma cells, activation of 
A

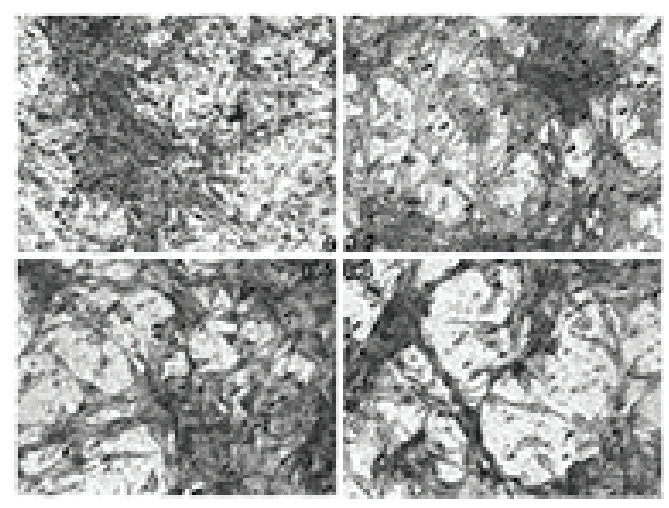

C

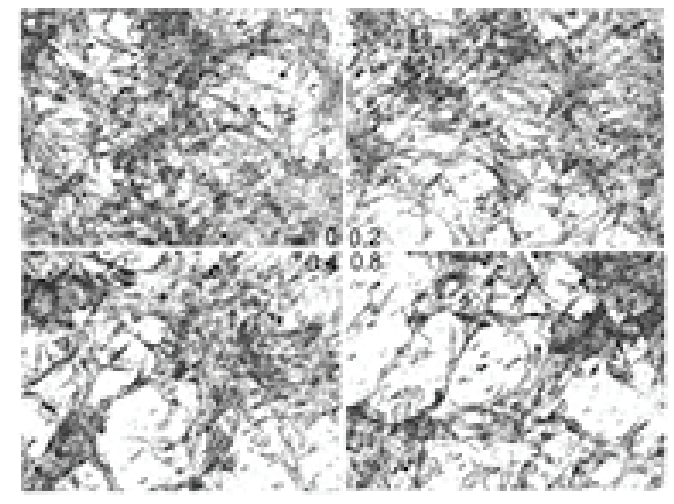

B

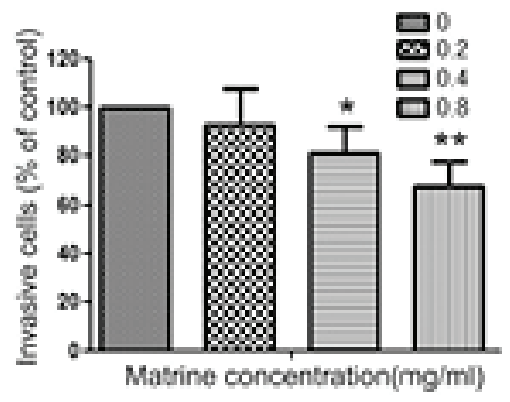

$\mathrm{D}$

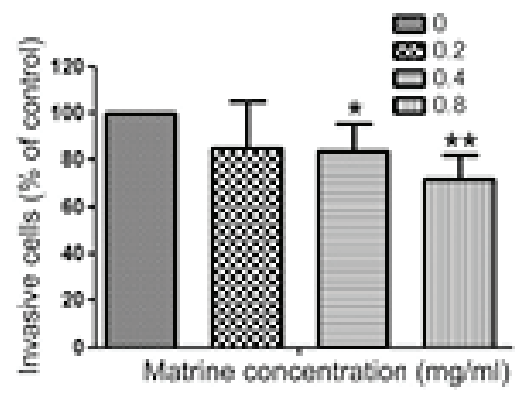

Figure 2. Effects of matrine on the migration and invasion of U87MG cells. (A) For the migration assay, cells were treated with various concentrations of matrine $(0 \mathrm{mg} / \mathrm{ml}$, top left; $0.2 \mathrm{mg} / \mathrm{ml}$, top right; $0.4 \mathrm{mg} / \mathrm{ml}$, bottom left; and $0.8 \mathrm{mg} / \mathrm{ml}$, bottom right) and plated onto the upper wells of the chamber. Fetal bovine serum (10\%) was added to the lower wells for $24 \mathrm{~h}$ to induce cell migration. After $24 \mathrm{~h}$, cells on the bottom side of the filter were fixed, stained and measured. Spontaneous cell migration in dimethyl sulfoxide was designated as the control. (B) The migration rate was expressed as a percentage of the control $(0 \mathrm{mg} / \mathrm{ml})$. (C) For the invasion assay, the chamber was coated with Matrigel, with all other steps the same as the migration assay. (D) The invasion rate was expressed as a percentage of the control $(0 \mathrm{mg} / \mathrm{ml})$. Values represent the means $\pm \mathrm{SD}$ of three independent experiments performed in triplicate. ${ }^{*} \mathrm{P}<0.05$ and ${ }^{* *} \mathrm{P}<0.01$ compared with control group.

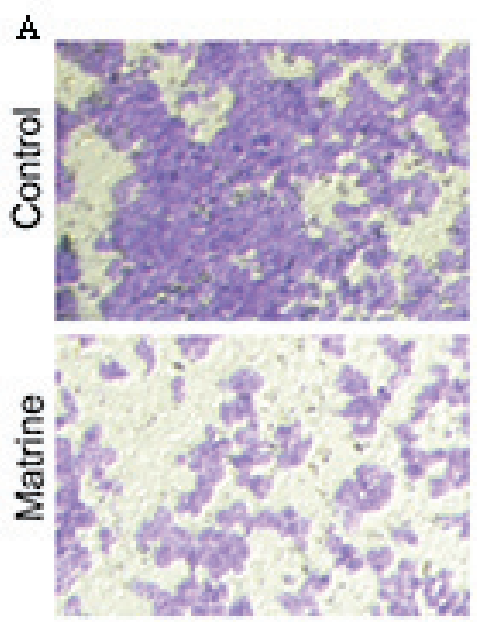

B

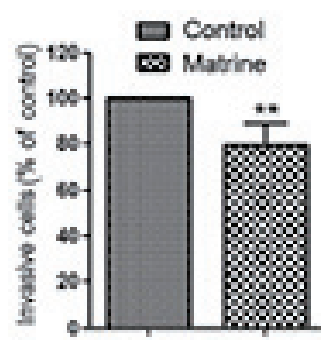

Figure 3. Effects of matrine on the invasion of U251MG cells. (A) For the invasion assay, the steps were the same as for the invasion assay on U87MG cells. (B) The invasion rate was expressed as a percentage of the control $(0 \mathrm{mg} / \mathrm{ml})$. Values represent the means $\pm \mathrm{SD}$ of three independent experiments performed in triplicate. ${ }^{* *} \mathrm{P}<0.01$ compared with control group.

p38 is required for the invasion process (12). Moreover, the mechanism is correlated with EMT; thus, we investigated the effect of matrine on p38 MAPK and AKT in U87MG cells. Western blot analysis revealed that matrine reduced the phosphorylation of p38 in a concentration-dependent manner
(Fig. 6A and B). At the same time, matrine inhibited the phosphorylation of AKT in a concentration-dependent manner (Fig. 6C and D).

\section{Discussion}

Glioma, in particular glioblastoma multiforme, with high morbidity and mortality rates, is a serious public health issue around the world (13). Matrine has been confirmed as a natural antitumor product in several types of cancer (14-17); however, the anti-metastatic effect of matrine and its associated mechanism(s) in glioma cells remain unclear. In this study we observed that matrine inhibited the mobility and invasive ability of glioma cells in vitro by regulating EMT via inhibition of the p38 MAPK and AKT pathways. To the best of our knowledge, this is the first scientific study of the anti-metastatic effect of matrine on glioma.

Transwell chambers (uncoated or coated with Matrigel) were employed to explore the effect of matrine on glioma cell migration and invasion. We demonstrated that matrine inhibited the migration and invasion of glioma cells at non-toxic doses (no more than $1 \mathrm{mg} / \mathrm{ml}$ ). These results revealed that the inhibition of invasion by matrine in U87MG cells was not due to cytotoxicity.

Tumor metastasis and recurrence is one of the most difficult challenges in the treatment of glioma patients. To complete the progression of metastasis, carcinoma cells must complete multiple 


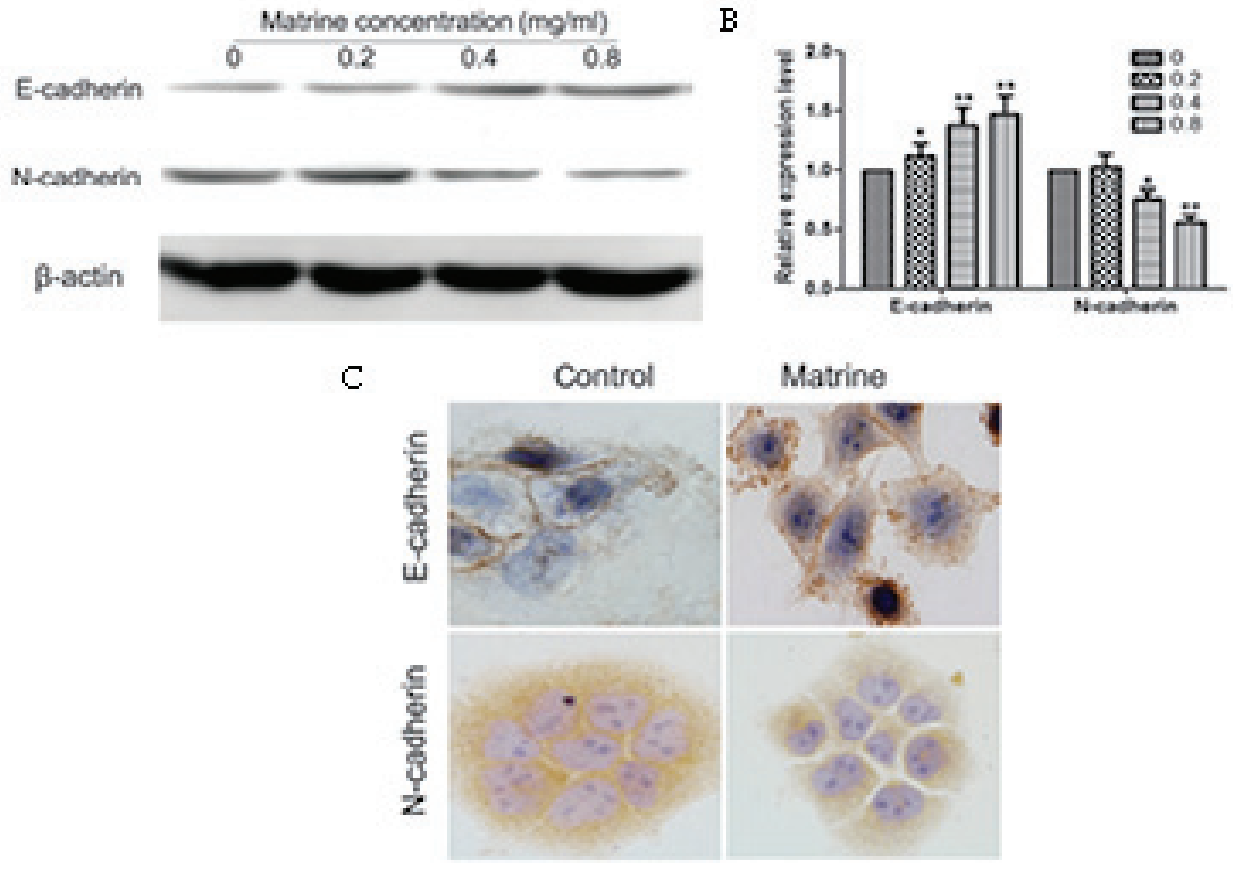

Figure 4. Matrine suppresses the expression of N-cadherin and increases the expression of E-cadherin in U87MG cells. (A) U87MG cells were treated with various concentrations of matrine $(0,0.2,0.4 \mathrm{and} 0.8 \mathrm{mg} / \mathrm{ml})$ for $24 \mathrm{~h}$ and then subjected to western blot analysis to assess the protein levels of E-cadherin and $\mathrm{N}$-cadherin. (B) Quantification of the protein levels of E-cadherin and $\mathrm{N}$-cadherin in U87MG cells. Values represent the means \pm SD of three independent experiments performed in triplicate. ${ }^{*} \mathrm{P}<0.05$ and ${ }^{* *} \mathrm{P}<0.01$ compared with control group. (C) Immunocytochemical staining for E-cadherin and $\mathrm{N}$-cadherin protein in U87MG cells with and without matrine treatment.

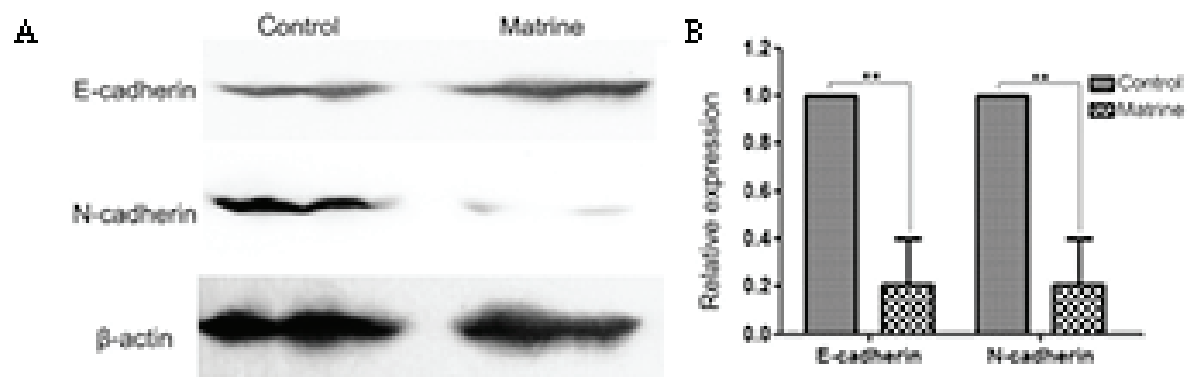

Figure 5. Matrine suppresses the expression of N-cadherin and increases the expression E-cadherin in U251MG cells. (A) U251MG cells were treated with various concentrations of matrine $(0$ and $0.8 \mathrm{mg} / \mathrm{ml})$ for $24 \mathrm{~h}$ and then subjected to western blot analysis to assess the protein levels of E-cadherin and N-cadherin. (B) Quantification of the protein levels of E-cadherin and N-cadherin in U251MG cells. Values represent the means \pm SD of three independent experiments performed in triplicate. ${ }^{* *} \mathrm{P}<0.01$ compared with control group.

A

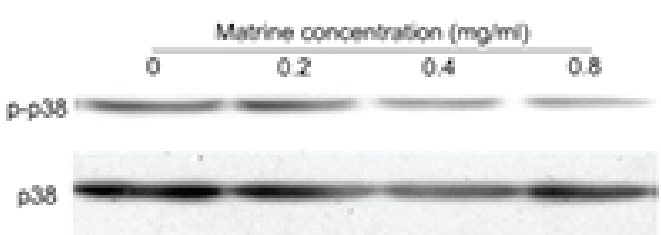

$C$

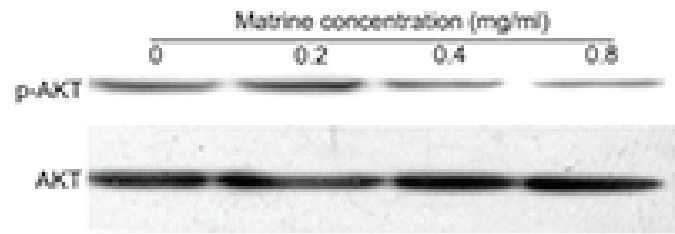

B
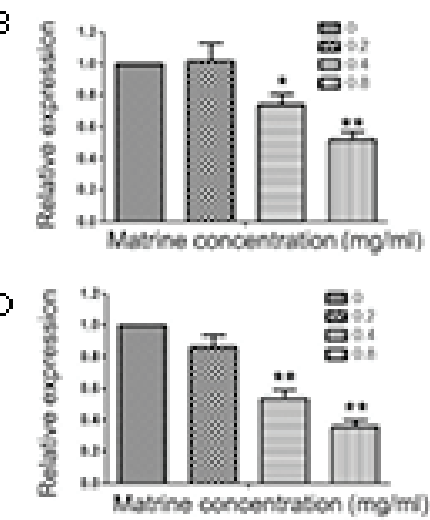

Figure 6. Effect of matrine on p38 MAPK and AKT pathways in U87MG cells. (A) Protein levels of p38 and p-p38. (B) Phosphorylation densities of p38 were digitally scanned. (C) Protein levels of AKT and p-AKT. (D) Phosphorylation densities of AKT were digitally scanned. Values represent the means \pm SD of three independent experiments performed in triplicate. ${ }^{*} \mathrm{P}<0.05$ and ${ }^{* *} \mathrm{P}<0.01$ compared with control group. 
distinct steps; this process has been termed the invasion-metastasis cascade, in which EMT plays a significant role (18). EMT induces epithelial cells to obtain mesenchymal markers and lose epithelial marker expression (19). As an epithelial molecular marker, E-cadherin is responsible for the establishment of the adherens junction, which forms a continuous adhesive belt below the apical surface (20). By mediating interactions with extracellular domains of E-cadherin molecules in adjacent cells, it forms intercellular junctions. Loss of E-cadherin by EMT results in the detachment of intercellular junctions and decreases adhesive force, simplifying the metastasis of carcinoma cells (20). The expression of mesenchymal markers, including $\mathrm{N}$-cadherin, increases. $\mathrm{N}$-cadherin is involved in the maintenance of microvessel stability and plays a role in blood vessel formation (21). In the present study, we observed that matrine increased the expression of E-cadherin and decreased the expression of $\mathrm{N}$-cadherin. The results suggest that the anti-metastatic effect of matrine on glioma is correlated with EMT.

The actual implementation of EMT is dependent on the concomitant activity of a variety of signal transduction pathways, including the MAPK and AKT signaling pathways (22). p38 MAPK plays a significant role in the induction of EMT by TGF- $\beta 1$ (23). p38 maintains E-cadherin expression by modulating TAK1-NF- $\kappa$ B during EMT in human primary mesothelial cells (24). In addition to MAPK signaling, AKT signaling plays a key role in inducing and maintaining EMT. Squamous cell carcinoma lines, expressing a constitutively active form of $\mathrm{PKB} / \mathrm{AKT}$, the most notable downstream effector of AKT signaling, underwent EMT, characterized by downregulation of the epithelial markers desmoplakin, E-cadherin and $\beta$-catenin, and upregulation of the mesenchymal marker vimentin $(25,26)$. Moreover, AKT signaling may also be activated by integrins and members of the Rho family of small GTPases that control cytoskeleton remodeling, a necessity during the morphogenic process of EMT $(27,28)$. In our study, we demonstrated that matrine effectively inhibited p38 MAPK and AKT signaling.

In conclusion, this study demonstrated the inhibitory effect of matrine on the invasion and metastatic capabilities of glioma cells. Furthermore, the decrease in the expression of $\mathrm{N}$-cadherin and the increase in the expression of E-cadherin induced by matrine is attributed to an inhibition of p38 MAPK and AKT signaling. This mechanism may contribute to the inhibition of invasion and metastasis in glioma cells by matrine. These findings reveal a new potential therapeutic application of matrine in anti-metastatic therapy for glioma.

\section{Acknowledgements}

This study was supported by grants from the Second Affiliated Hospital, School of Medicine, Xi'an Jiaotong University [YJ (QN) 201219], and Science and Technology Projects in Shaanxi Province (2013k13-01-09).

\section{References}

1. DeAngelis LM: Brain tumors. N Engl J Med 344: 114-123, 2001

2. Giese A, Rief MD, Loo MA and Berens ME: Determinants of human astrocytoma migration. Cancer Res 54: 3897-3904, 1994.

3. Giese A, Bjerkvig R, Berens ME and Westphal M: Cost of migration: invasion of malignant gliomas and implications for treatment. J Clin Oncol 21: 1624-1636, 2003.
4. Jansen M, Yip S and Louis DN: Molecular pathology in adult gliomas: diagnostic, prognostic, and predictive markers. Lancet Neurol 9: 717-726, 2010

5. Li LQ, Li XL, Wang L, et al: Matrine inhibits breast cancer growth via miR-21/PTEN/Akt pathway in MCF-7 cells. Cell Physiol Biochem 30: 631-641, 2012.

6. Zhang Z, Wang $\mathrm{X}, \mathrm{Wu} \mathrm{W}$, et al: Effects of matrine on proliferation and apoptosis in gallbladder carcinoma cells (GBC-SD). Phytother Res 26: 932-937, 2012.

7. Zhang S, Zhang Y, Zhuang Y, et al: Matrine induces apoptosis in human acute myeloid leukemia cells via the mitochondrial pathway and Akt inactivation. PLoS One 7: e46853, 2012.

8. Wang Z, Zhang J, Wang Y, et al: Matrine, a novel autophagy inhibitor, blocks trafficking and the proteolytic activation of lysosomal proteases. Carcinogenesis 34: 128-138, 2013.

9. Chen K, Zhang S, Ji Y, et al: Baicalein inhibits the invasion and metastatic capabilities of hepatocellular carcinoma cells via down-regulation of the ERK pathway. PLoS One 8: e72927, 2013.

10. Yang SF, Yang WE, Kuo WH, Chang HR, Chu SC and Hsieh YS: Antimetastatic potentials of flavones on oral cancer cell via an inhibition of matrix-degrading proteases. Arch Oral Biol 53: 287-294, 2008

11. Gao Q, Liu W, Cai J, et al: EphB2 promotes cervical cancer progression by inducing epithelial-mesenchymal transition. Hum Pathol 45: 372-381, 2014.

12. Demuth T, Reavie LB, Rennert JL, et al: MAP-ing glioma invasion: mitogen-activated protein kinase kinase 3 and $\mathrm{p} 38$ drive glioma invasion and progression and predict patient survival. Mol Cancer Ther 6: 1212-1222, 2007.

13. Wang ZS, Luo P, Dai SH, Liu ZB, Zheng XR and Chen T: Salvianolic acid B induces apoptosis in human glioma U87 cells through p38-mediated ROS generation. Cell Mol Neurobiol 33: 921-928, 2013.

14. Mondal S, Bandyopadhyay S, Ghosh MK, Mukhopadhyay S, Roy S and Mandal C: Natural products: promising resources for cancer drug discovery. Anticancer Agents Med Chem 12: 49-75, 2012.

15. Chandrashekar N, Selvamani A, Subramanian R, Pandi A and Thiruvengadam D: Baicalein inhibits pulmonary carcinogenesis-associated inflammation and interferes with COX-2, MMP-2 and MMP-9 expressions in-vivo. Toxicol Appl Pharmacol 261: 10-21, 2012.

16. Wu B, Li J, Huang D, et al: Baicalein mediates inhibition of migration and invasiveness of skin carcinoma through Ezrin in A431 cells. BMC Cancer 11: 527, 2011.

17. Wang ZD, Huang C, Li ZF, et al: Chrysanthemum indicum ethanolic extract inhibits invasion of hepatocellular carcinoma via regulation of MMP/TIMP balance as therapeutic target. Oncol Rep 23: 413-421, 2010.

18. Yilmaz M and Christofori G: EMT, the cytoskeleton, and cancer cell invasion. Cancer Metastasis Rev 28: 15-33, 2009.

19. Xu J, Lamouille S and Derynck R: TGF-beta-induced epithelial to mesenchymal transition. Cell Res 19: 156-172, 2009.

20. Christiansen JJ and Rajasekaran AK: Reassessing epithelial to mesenchymal transition as a prerequisite for carcinoma invasion and metastasis. Cancer Res 66: 8319-8326, 2006.

21. Blaschuk OW and Devemy E: Cadherins as novel targets for anti-cancer therapy. Eur J Pharmacol 625: 195-198, 2009.

22. Tiwari N, Gheldof A, Tatari M and Christofori G: EMT as the ultimate survival mechanism of cancer cells. Semin Cancer Biol 22: 194-207, 2012.

23. Chen HH, Zhou XL, Shi YL and Yang J: Roles of p38 MAPK and JNK in TGF-beta1-induced human alveolar epithelial to mesenchymal transition. Arch Med Res 44: 93-98, 2013.

24. Strippoli R, Benedicto I, Foronda M, et al: p38 maintains E-cadherin expression by modulating TAK1-NF-kappaB during epithelial-to-mesenchymal transition. J Cell Sci 123: 4321-4331, 2010.

25. Wei J, Li Z, Chen W, et al: AEG-1 participates in TGF-beta1-induced EMT through p38 MAPK activation. Cell Biol Int 37: 1016-1021, 2013.

26. Grille SJ, Bellacosa A, Upson J, et al: The protein kinase Akt induces epithelial mesenchymal transition and promotes enhanced motility and invasiveness of squamous cell carcinoma lines. Cancer Res 63: 2172-2178, 2003.

27. Zamir E and Geiger B: Molecular complexity and dynamics of cell-matrix adhesions. J Cell Sci 114: 3583-3590, 2001.

28. Xia N, Thodeti CK, Hunt TP, et al: Directional control of cell motility through focal adhesion positioning and spatial control of Rac activation. FASEB J 22: 1649-1659, 2008. 